\title{
Universal force correlations in an RNA-DNA unzipping experiment
}

\author{
Kay Jörg Wiese $\odot,{ }^{1}$ Mathilde Bercy, ${ }^{2}$ Lena Melkonyan, ${ }^{2}$ and Thierry Bizebard ${ }^{3}$ \\ ${ }^{1}$ Laboratoire de Physique de l'École Normale Supérieure, ENS, Université PSL, CNRS, Sorbonne Université, \\ Université Paris-Diderot, Sorbonne Paris Cité, 24 rue Lhomond, 75005 Paris, France \\ ${ }^{2}$ UMR 7615, Ecole Supérieure de Physique et Chimie Industrielles de la Ville de Paris, Paris, France \\ ${ }^{3}$ UMR 8104, Institut Cochin, 22 rue Méchain, 75014 Paris, France
}

(Received 9 September 2019; accepted 24 November 2020; published 17 December 2020)

\begin{abstract}
We study unzipping of a complementary RNA-DNA helix applied to an external force, focusing on the force-force correlations. While at the microscopic level these are given by the sequence, the experiment measures effective, macroscopic correlations. The latter are sequence independent, i.e., universal, and constitute the central object of the underlying field theory of disordered systems. Comparing field-theory predictions and the exact solution of a one-dimensional toy model with the experiment, we find an excellent agreement, confirming fundamental theoretical concepts via biologically inspired experiments.
\end{abstract}

DOI: 10.1103/PhysRevResearch.2.043385

\section{INTRODUCTION}

The amount of biological data is growing steadily, reaching about $2.5 \times 10^{16}$ Bytes in 2015 [1], on par with astronomy, youtube, and Twitter. An important question is what can be learned from these data, and what cannot? Depending on their specialization, scientists usually ask different, and seemingly unrelated questions. Here we study unzipping in the peeling mode [2] of a complementary RNA-DNA double strand, using a sequence obtained from ribosomal RNA. As shown in Fig. 1, at one end the double helix is attached with its both strands to a bead, whereas on the other end only the DNA strand is. Pulling on the beads with an optical tweezer [3] the RNA strand peels off. What is measured is the force-extension curve, of which an example is given in Fig. 2.

Rather complementary questions can now be asked:

(i) What can one learn about the specific biological system?

(ii) Are there observables which are independent of the chosen nucleotide sequence, thus universal?

(iii) How does understanding the universal signal help to analyze the biological system? What limitations does it impose?

The first question is at the origin and design of the experiment $[4,5,7]$, which aims at understanding assembly of the large subunit of the ribosome, and where all experimental parameters can be found. Here we address the second question, aiming at understanding its universal, sequence-independent properties. This allows us to address the third question, helping to better extract biologically relevant data.

Published by the American Physical Society under the terms of the Creative Commons Attribution 4.0 International license. Further distribution of this work must maintain attribution to the author(s) and the published article's title, journal citation, and DOI.
Consider the force-extension curve in Fig. 2. Applying no force, the RNA-DNA double strand is in an equilibrated coiled state, with its end-to-end distance being roughly $0.8 \mu \mathrm{m}$. Since the beads are sitting in an optical trap, their distance, or more specifically the distance $w$ between the two minima of the trap, is the control parameter. Increasing $w$, the RNADNA double strand is stretched, reflected in an increase in the measured force $F$. Finally part of the RNA sequence peels off [8], leading to a first drop in the force-extension curve. Increasing $w$ further leads to more force drops resulting in an almost constant force. This plateau regime is marked in red in Fig. 2. Increasing $w$ further, peeling can no longer reduce the force, and the latter increases again, eventually leading to the breakage of the DNA molecule (not shown). If instead of $w$ the applied force $F$ were controlled, as in experiments with magnetic tweezers $[9,10]$, a phase transition at $F_{\mathrm{c}}$ could be observed between a closed and an open state [11].

The aim of this article is to analyze the force fluctuations on the plateau, i.e., the sawtooth shaped signal on top of the critical force. This kind of signal is frequent in nature, and at the heart of the so-called depinning transition: It arises in a plethora of situations: Barkhausen noise in magnets $[12,13]$

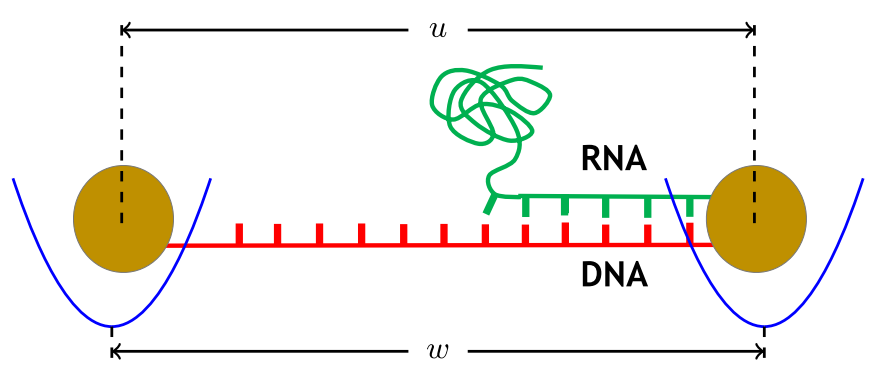

FIG. 1. Peeling of an RNA-DNA double strand. The RNA sequence is from subunit $23 \mathrm{~S}$ of the ribosome in E. Coli, prolonged to attach the beads (with a much larger radius than drawn here). The DNA sequence is its complement. Drawing not to scale. 


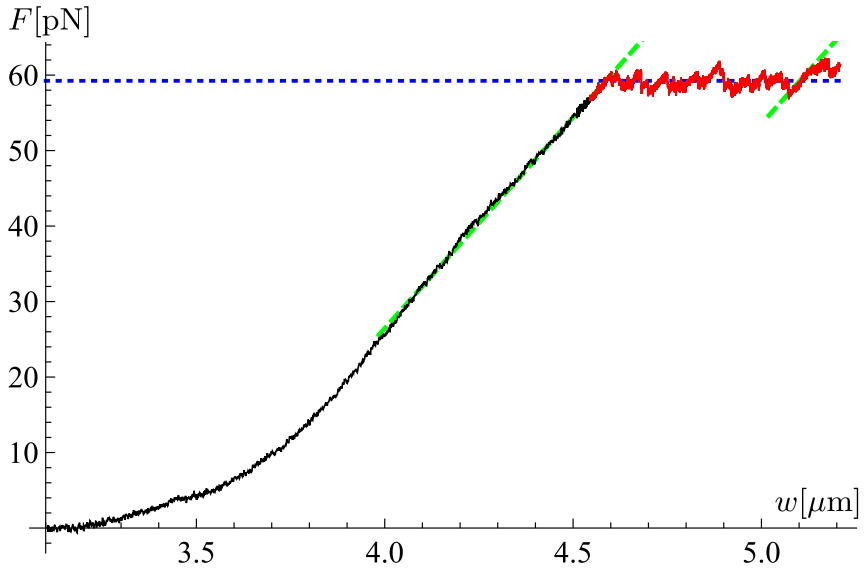

FIG. 2. A sample force-extension curve. For the data analysis we only use the last part of the curve, the plateau (in red). On this plateau, the force fluctuates around its critical value of about $60 \mathrm{pN}$. The extension $w$ starts at $3 \mu \mathrm{m}$, which is the sum of the unstretched molecule plus twice the radius of the beads $(2 \times 1 \mu \mathrm{m})$. The effective stiffness $m^{2}$ in Eq. (1) is estimated from the slope of the green dashed lines as $m^{2}=55 \pm 5 \mathrm{pN} / \mu \mathrm{m}$ at the beginning of the plateau, which remains at least approximately the same at the end of the plateau. The driving velocity is about $7 \mathrm{~nm} / \mathrm{s}$, corresponding to 42 nucleotides $/ \mathrm{s}$ as in the cell [6].

(audible as the rustle in old-style telephones), depinning of a contactline [14] (the line where coffee and air meet in a cup, or in drops on a windshield), earthquakes [15], vortices in high-temperature superconductors [16], to name a few. The largest such system on earth is the movement of tectonic plates in the outer crust of the earth, where the resulting force drops are earthquakes. The smallest system the authors are aware of is the peeling experiment studied here. Yet, all these systems have a very similar phenomenology: In each case, a control parameter $w$ is increased, leading to an increase in tension of the elastic object, released via a succession of force drops. Being omnipresent, many theoretical models and mechanisms have been proposed for this depinning transition, starting from the chaos induced in the Burridge-Knopoff model of 1967 [17], over toy models for magnets $[18,19]$, to sophisticated field theoretic work using functional RG [20-25]. Today it is understood that the minimal ingredients are

(i) a random force (the disorder),

(ii) an elastic coupling to an external control parameter,

(iii) an overdamped dynamics.

In the experiment considered here, the random force comes from the seemingly random RNA sequence of the ribosome [26]. The elastic coupling to an external control parameter is given by the beads attached to the ends of the strands sitting in the harmonic traps with distance $w$. Finally, an overdamped dynamics is typical for small systems immersed into a solvent, where inertia plays a negligible role.

\section{THEORY}

The measured force can be expressed as [27]

$$
F=m^{2}(w-u)
$$

where $w$ is the distance between the two traps, and $u$ is the distance between the two beads, see Fig. 1. This corresponds to an energy $\mathcal{E}=\frac{m^{2}}{2}(w-u)^{2}$ where $m^{2}$ is the combined strength of the traps, the elasticity of the partially unzipped double strand, and the attached single strand. What is measured in the experiment is the force given in Eq. (1). An example is shown in Fig. 2. Upon increasing the trap distance $w$, the force first increases until it reaches a plateau regime where it is almost constant, $\langle F\rangle \approx F_{\mathrm{c}}=60 \mathrm{pN}$. We are interested in the correlations of the force fluctuations in the plateau regime (red in Fig. 2), i.e., the connected expectations

$$
\begin{aligned}
\Delta\left(w, w^{\prime}\right) & :=\left\langle F(w) F\left(w^{\prime}\right)\right\rangle^{\mathrm{c}} \\
& \equiv\left\langle[F(w)-\langle F(w)\rangle]\left[F\left(w^{\prime}\right)-\left\langle F\left(w^{\prime}\right)\right\rangle\right]\right\rangle .
\end{aligned}
$$

Here $w$ and $w^{\prime}$ are two distinct values of $w$ in Fig. 2. Since $\Delta\left(w, w^{\prime}\right)$ only depends on the difference $w-w^{\prime}$ we can improve the disorder average (average over experiments) by an additional translational average.

Let us start with an exact solution for a toy model [28], namely a particle dragged through a disordered force landscape. To apply the results of Ref. [28] one needs to specify the distribution of forces $F$. Since the microscopic forces can be thought of as sums of random variables (neighboring monomers act together to generate these random forces), and assuming the central-limit theorem applies, forces are Gauss distributed, which in the terminology of [28] leads to the Gumbell universality class of extreme-value statistics, with correlator

$$
\begin{aligned}
\Delta(w) & =m^{4} \rho_{m}^{2} \Delta_{\text {Gumbell }}\left(w / \rho_{m}\right), \\
\Delta_{\text {Gumbell }}(x) & :=\frac{x^{2}}{2}+\operatorname{Li}_{2}\left(1-e^{|x|}\right)+\frac{\pi^{2}}{6}, \\
\rho_{m} & =\frac{1}{m^{2} \sqrt{2 \ln \left(m^{-2}\right)}} .
\end{aligned}
$$

As shown below in Sec. III and in Fig. 5, experimental data and theory agree well.

While it is gratifying to have a theoretical prediction verified by an experiment, the implications here are much deeper, as the function $\Delta(w)$ is the central object of the field theory of disordered elastic manifolds of inner dimension $d$ (here $d=$ $0)$, e.g., a magnetic domain wall in a bulk magnet $(d=2)$, or a contact line $(d=1)$. These systems are governed by an equation of motion for the domain wall or line $u$, parametrized by an internal coordinate $x$ and time $t$,

$$
\partial_{t} u(x, t)=\nabla^{2} u(x, t)+m^{2}[w-u(x, t)]+F(x, u(x, t)) .
$$

The force $F(w)$ is the force acting on the center of mass. Then the renormalized force-force correlator [29-31]

$$
\Delta\left(w-w^{\prime}\right):=\frac{1}{L^{d}}\left\langle F(w) F\left(w^{\prime}\right)\right\rangle^{\mathrm{c}},
$$

where $L$ is the linear size of the system, and $L^{d}$ its volume, can be obtained from field theory [20,21,24,31], based on the equation of motion (6). Field theory is a central tool in theoretical physics [32], with applications ranging from elementary particle physics [33] to the fluctuations observed around the critical point in liquid-gas transitions [34]. In all these cases, a 


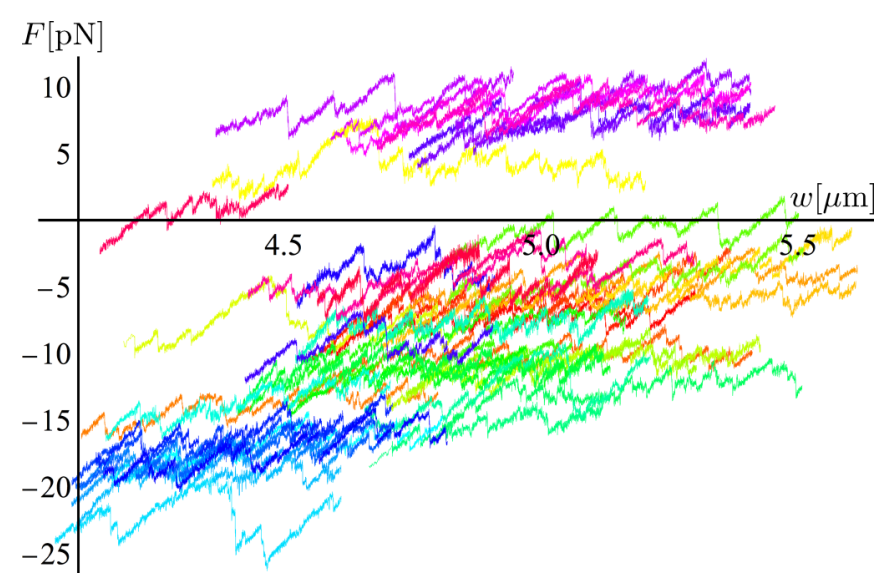

FIG. 3. Force-extension curves restricted to the plateau region for one of our batches with 47 data sets. Curves are randomly displaced for better visualization.

set of flow equations for a finite number of coupling constants is derived. These methods fail for disordered systems as those given by Eq. (6). A way out was found by realizing that the flow for the coupling constants has to be generalized to flow for a function. This is known as the functional renormalization group (FRG). The flow equations take the form

$$
\partial_{\ell} \Delta(w)=-\frac{d^{2}}{d w^{2}} \frac{1}{2}[\Delta(w)-\Delta(0)]^{2}+\cdots,
$$

where the omitted terms are higher-order corrections (technically higher-loop terms [23-25,32]), equivalent to an expansion in $\varepsilon=4-d$ (with $d$ the dimension of the object). What came as a surprise was the realization that $\Delta(w)$ appearing in Eq. (8), when integrated from a microscopic scale to the length scale $\ell \equiv 1 / m$, is the disorder-force correlator measured via Eq. (7) [29-31]. Measuring $\Delta(w)$ is thus a key test $[14,35,36]$ for the field theory of disordered systems. The solution to Eq. (8) (leading order in the expansion parameter $\varepsilon)$ reads

$$
\begin{aligned}
\Delta(w) & =\mathcal{A} \Delta_{\mathrm{FT}}(w / \rho), \\
\Delta_{\mathrm{FT}}(x) & =-W\left[-\exp \left(-\frac{x^{2}}{2}-1\right)\right],
\end{aligned}
$$

where $\mathcal{A}$ and $\rho$ are nonuniversal constants, and the product$\log W(z)$ is the principal solution for $w$ in $z=w e^{w}$. Field theory also applies to the experiment described above, which has (internal) dimension $d=0$ (the single degree of freedom is the number of the last unpeeled monomer). As $\varepsilon=4$ is large, we expect Eqs. (9) and (10) to give a decent approximation for the experiment, but also to show differences. This is confirmed below, see Sec. III and Fig. 5.

\section{DATA ANALYSIS AND UNIVERSAL SIGNAL}

We measure the force-extension curve in an RNA-DNAunzipping experiment $[4,5,7]$, retaining from the forceextension curve shown in Fig. 2 only the plateau part (in red). This experiment was repeated 163 times. From one of the batches with 47 data sets, we show the retained plateaux in Fig. 3. In order to minimize statistical errors, we measure the

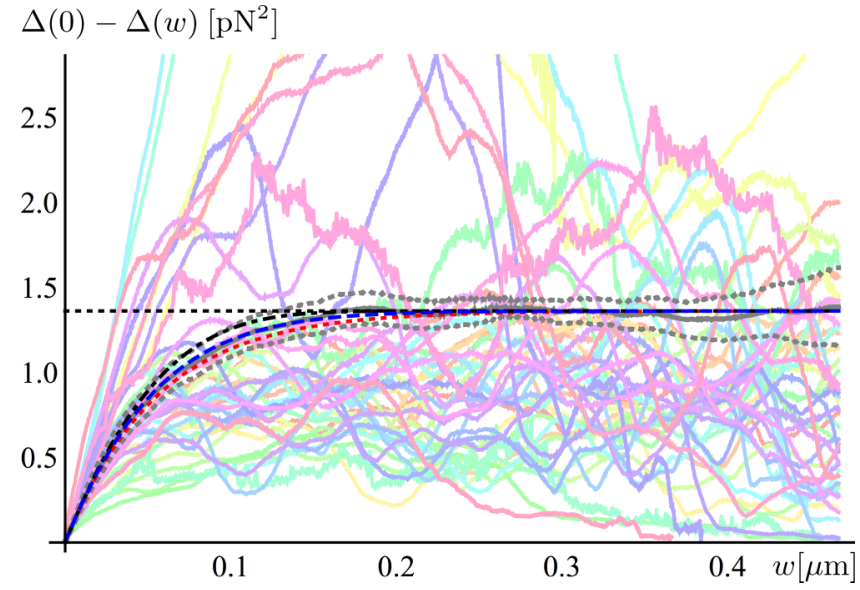

FIG. 4. Estimation of $\Delta(0)-\Delta(w)$ from one of our batches with 47 data sets, compared to three theoretical curves: pure exponential decay (red), 1-loop FRG (black dot-dashed), and toy model (blue dashed)

combination $\Delta(0)-\Delta(w)=\frac{1}{2}\left\langle[F(u+w)-F(u)]^{2}\right\rangle^{c}$. This average is more stable experimentally, since there seems to be a small drift in the data (visible in Fig. 3); the latter may be induced by a slightly diminishing effective stiffness $m^{2}$ upon opening the strands, even though this is invisible in Fig. 2.

In Fig. 4 we show the combination $\Delta(0)-\Delta(w)$ as defined by Eq. (7), for each of the force-extension curves of Fig. 3, with the shaded colors identical to those of Fig. 3. Strong statistical fluctuations are visible. Their mean, in solid gray, is compared to three theoretical curves: The leadingorder field theory result (10) (black dot-dashed line), the Gumbell result (3) (blue, dashed line), and an exponentially decaying function (red, dotted line). There are two unknown scales, equivalent to a rescaling of $w$ and $\Delta$. Since the slope at the origin can be measured precisely, we rescale all theoretical functions to have the same slope [37]. The remaining parameter is the behavior of $\Delta(0)-\Delta(w)$ for large $w$, which is adjusted visually. In dotted gray we show our estimates of the absolute error bars, obtained by resampling, as explained in Appendix A.

The result (of these partial data) favors the theoretical prediction (3), while the estimated error bars are seemingly rather large. The reason for the latter is that the main statistical fluctuations come from the amplitude multiplying $\Delta(w)$. Measuring the statistical error of $\Delta(w) / \Delta(0)$ estimates the error of the shape only. It is further reduced by using all our data, and is indicated by the green shaded region on our final curve in Fig. 5. The agreement of the theory and the experimental data is excellent, better than expected from the single measurements of Fig. 4. This strongly indicates that the universal physics behind the depinning transition is robust.

\section{SCALES AND INTERPRETATION}

Our final result for $\Delta(w)$, given by the gray solid line in Fig. 5, is in remarkable agreement with the analytical result (3). What does this mean? Consider Fig. 2, where the force grows linearly, interrupted by sudden drops of size $\delta F$. One can show [38] that the derivative of the function $\Delta(w)$ at the 


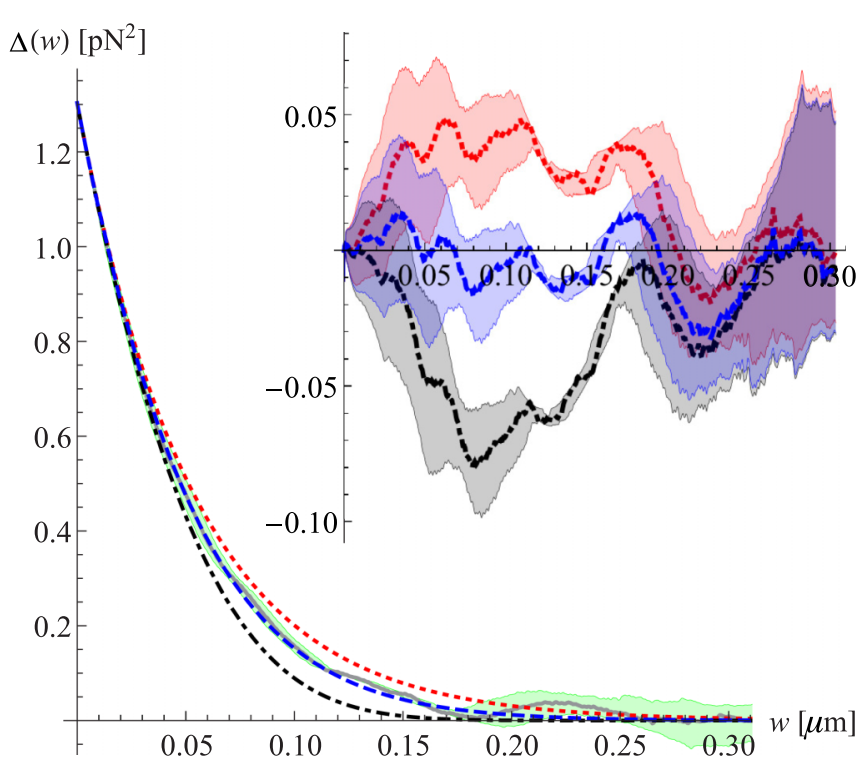

FIG. 5. Measurements of $\Delta(w)$ (in gray), with $1-\sigma$ error bars (green shaded), compared to three theoretical curves: pure exponential decay (dotted red line), 1-loop FRG (black dot-dashed line), and toy model (blue dashed line). Inset: Theoretical curves with the data subtracted (same color code). The blue curve is the closest to the data.

origin is related to a moment ratio of force drops [39]

$$
\left|\Delta^{\prime}\left(0^{+}\right)\right|=m^{2} \delta F_{m}, \quad \delta F_{m}=\frac{\left\langle\delta F^{2}\right\rangle}{2\langle\delta F\rangle} .
$$

Our experiments yield $m^{2}=55 \pm 5 \mathrm{pN} / \mu \mathrm{m}$ (see Fig. 1) leading to $\delta F_{m}=0.43 \pm 0.05 \mathrm{pN}$, and to a correlation length $\xi=0.055 \pm 0.005 \mu \mathrm{m} \simeq 186$ base pairs. This is roughly consistent with the nine force drops identifiable in Fig. 2. The driving velocity was varied from 5 to $7 \mathrm{~nm} / \mathrm{s}$, where no statistically significant difference was observed for $\Delta(w)$.

These measurements indicate a serious challenge for peeling experiments using optical tweezers: As force-force correlations decay on a scale of about 200 bases, which is about $1 / 15$ of the length of the ribosomal RNA, events can be resolved with that resolution. As Eq. (5) shows, this resolution increases with the stiffness $m^{2}$. The key to a high resolution is thus a stiff construction and a well-aligned trap: If the trap is not optimally aligned, showing the critical force at a say $20 \%$ smaller value, the resolution suffers according to Eqs. (3)-(5) by an even larger amount. Another possibility to increase the stiffness is to use shorter constructions.

Our system maximizes force differences, and thus the measured signal $\Delta(w)$, as the two possible parings CG and AT/AU with different binding energies appear almost in the same proportion [4]. The binding enthalpy $\delta \mathcal{H}$ per hydrogen bond is about $2 \mathrm{kcal} / \mathrm{mol}$ [40], which gives $5 \mathrm{kcal} / \mathrm{mol}$ on average for the two possible pairings. This is augmented by stacking energies between the DNA and RNA strand to 9.1 $\mathrm{kcal} / \mathrm{mol}$ [41]. The mean free energies $\delta \mathcal{G}$ are $1.5 \mathrm{kcal} / \mathrm{mol}$ [41]. The difference in the number of hydrogen bonds gives binding energy fluctuations of $1 \mathrm{kcal} / \mathrm{mol}$, which is $11 \%$ of the total binding energy. The measured force $F_{\mathrm{c}}$ on the plateau is $60 \mathrm{pN}$, which leads to a microscopic force fluctuation of $d F=6.6 \mathrm{pN}$. In the experiment we observe $d F=1.14 \mathrm{pN}$. Why is this value so low? If we use the correlation length of 186 base pairs, equate it with $\rho$ in Eqs. (3)-(5), this predicts $m^{2}=1.5 \times 10^{-3}$, and $\ln \left(m^{-2}\right)=6.5$. This reduces $d F$ from 6.6 to $2.6 \mathrm{pN}$, about 2 times larger than in the experiment. Several factors may contribute to a further reduction: thermal fluctuations, and the appearance of base pairs in the free-energy estimates [41]. Finally, we did not discard "bad" samples, i.e., samples with vanishing signal, since any filtering risks introducing a systematic bias. While this does not change the shape of $\Delta(w)$, it underestimates its amplitude. Thus our measurements agree qualitatively with this crude estimate, and more should not be expected.

The function $\Delta(w)$ was also measured in contact-line depinning [14], and studied numerically for magnetic domain walls $[35,36]$. For all these systems thermal fluctuations play no role. In our experiment, thermal fluctuations are clearly visible, both in an additional white noise for each data point, as in a reduction of $\delta \mathcal{H} \approx 9.1 \mathrm{kcal} / \mathrm{mol}$ to $\delta \mathcal{G} \approx 1.5 \mathrm{kcal} / \mathrm{mol}$. Expectations in the literature are that thermal fluctuations may round the cusp [31,42], a feature not visible here. How can this be reconciled? There are several distinct entropic contributions: Conformational entropy of unbound strands, a higher entropy for the salt ions for unbound strands, and binding entropy, as a bond can either be open or closed. The first two ones are large and reduce $\delta \mathcal{H}$ quite substantially to $\delta \mathcal{G}$; the last one is small: as $k_{\mathrm{B}} T \approx 0.6 \mathrm{kcal} / \mathrm{mol}$, the probability that a bond is broken by thermal fluctuations is $p_{T}=e^{-\delta \mathcal{G} / k_{\mathrm{B}} T}$, with values ranging from $8 \times 10^{-3}$ to 0.7 using the binding free energies $\delta \mathcal{G}$ of [41]. Thus at most a few bonds can be opened by thermal fluctuations, and using Eqs. (3) and (10) based on zero-temperature depinning is justified.

One should be able to extract $\Delta(w)$ also from the unzipping [2] of a hairpin. Interestingly, experiments report that the scaling of Eq. (5) is replaced by $\rho_{m} \sim m^{-4 / 3}$ [43], a clear signature of a different universality class, namely "random-field" disorder in equilibrium [44]. This scenario is possible through the much stronger effective stiffness $m^{2}$ there. Equilibrium is observed experimentally through a vanishing hysteresis curve.

While the sequence used in the experiments is extracted from ribosomal RNA, thus is not random, the measured function $\Delta(w)$ agrees to a good precision with that obtained for a random sequence. In conclusion, universal physics can emerge even in a specific biological system, and for a relatively short sequence.

\section{APPENDIX A: DATA ANALYSIS AND ERROR ESTIMATES}

Protocol and error estimates: Define for a data-set $\mathcal{D}_{i}$, with $i=1, \ldots, n$ and $n$ the total number of force-extension curves, the set average

$$
\begin{aligned}
N_{i}(w) & :=\sum_{u \in \mathcal{D}_{i}}, \\
Q_{i}(w) & :=\frac{1}{N_{i}(w)} \sum_{u \in \mathcal{D}_{i}}[F(u+w)-F(u)]^{2}, \\
M_{i}(w) & :=\frac{1}{N_{i}(w)} \sum_{u \in \mathcal{D}_{i}}[F(u+w)-F(u)],
\end{aligned}
$$




$$
Q_{i}^{\mathrm{c}}(w):=Q_{i}(w)-M_{i}(w)^{2} .
$$

The above sums run over all values $u$, for which exists a pair $F(u+w)$ and $F(u) ; N_{i}(w)$ is the number of such pairs. Our best estimate for the force-force correlator then is

$$
\left\langle[F(u+w)-F(u)]^{2}\right\rangle^{\mathrm{c}}=\frac{\sum_{i} Q_{i}^{\mathrm{c}}(w) N_{i}(w)}{\sum_{i} N_{i}(w)} .
$$

The fluctuations of the data shown in Fig. 4 are very large, making error estimates difficult. We used a statistical resampling technique: Randomly divide all data-sets $\mathcal{D}_{i}$ into two parts, $\mathcal{P}_{1}$ and $\mathcal{P}_{2}$. Define

$$
\begin{aligned}
& N_{\mathcal{P}_{1}}(w):=\sum_{i \in \mathcal{P}_{1}} N_{i}(w), \\
& Q_{\mathcal{P}_{1}}^{\mathrm{c}}(w):=\frac{1}{N_{\mathcal{P}_{1}}(w)} \sum_{i \in \mathcal{P}_{1}} Q_{i}^{\mathrm{c}}(w) N_{i}(w) .
\end{aligned}
$$

A similar definition holds for $\mathcal{P}_{2}$. Then for each $w$ measure the variance of the partial means $Q_{\mathcal{P}_{1}}^{\mathrm{c}}(w)$ and $Q_{\mathcal{P}_{2}}^{\mathrm{c}}(w)$. Finally, average over all partitions $\Pi_{i},\{1, \ldots, n\} \rightarrow \mathcal{P}_{1}, \mathcal{P}_{2}$. In practice, it is enough to take $N_{\mathrm{p}}=100$ random partitions. The error estimate then is

$$
\begin{aligned}
\sigma^{2}(w) & :=\frac{1}{N_{\mathrm{p}}} \sum_{\Pi_{i}}\left\langle\frac{1}{2} \sum_{k=1}^{2}\left[Q_{\mathcal{P}_{k}\left(\Pi_{i}\right)}^{\mathrm{c}}(w)-Q^{\mathrm{c}}(w)\right]^{2}\right\rangle, \\
N_{\mathrm{p}} & :=\sum_{\Pi_{i}} .
\end{aligned}
$$

We can also define the set of all $2 N_{\mathrm{p}}$ partial means,

$$
\mathcal{A}(w):=\bigcup_{\Pi_{i}} \bigcup_{k=1,2} Q_{\mathcal{P}_{k}\left(\Pi_{i}\right)}^{\mathrm{c}}(w) .
$$

We find that our analysis is consistent with

$$
\operatorname{var}(\mathcal{A}(w)) \approx \sigma^{2}(w) .
$$

These error estimates are absolute errors, presented in Fig. 4. To obtain the error estimate given in Fig. 5, the partial means (A10) where rescaled such that their $w$ integrals equal the $w$ integral over all samples. This takes out amplitude fluctuations, reducing the errors to errors of the shape.

\section{APPENDIX B: CHECK ON TEST DATA}

We generated test data according to the following protocol: For each real data set, sample an Ornstein-Uhlenbeck process of the same length, with mean $F_{m}=F_{c}$, variance $\Delta(0)$, and correlation length $\xi$ as measured. This is achieved by the stochastic process

$$
\begin{aligned}
F(w+\delta w) & =F(w)+\zeta(w) \sqrt{\frac{\Delta(0)}{\xi}}+\frac{F_{m}-F(w)}{\xi}, \\
\left\langle\zeta(w) \zeta\left(w^{\prime}\right)\right\rangle & =\delta_{w, w^{\prime}} .
\end{aligned}
$$

This gives a first set of test data. For a second set we add an additional white noise in the $x$ direction, with $\delta x \in\{-1,0,1\}$, in units of the resolution of the measuring machine. For a third set we added a Gauss distribution of mean zero and width 1 to the force signal. By construction, these test data are exponentially correlated:

$$
\left\langle F(w) F\left(w^{\prime}\right)\right\rangle^{\mathrm{c}}=\Delta(0) e^{-\left|w-w^{\prime}\right| / \xi},
$$

with additional noise for sets 2 and 3. They should thus approach the red dotted curve of Fig. 5. This is indeed observed, with an appropriate estimate for the error bars.
[1] Z. Stephens, S. Lee, F. Faghri, R. Campbell, C. Zhai, M. Efron, R. Iyer, M. Schatz, S. Sinha, and G. Robinson, Big data: Astronomical or genomical? PLOS Biol. 13, e1002195 (2015).

[2] In the literature, the word peeling is used for the setup of Fig. 1, where forces act along the helical axis from opposite extremities of a duplex, and one of the two strands peels off. Unzipping denotes an alternative setup where the right bead of Fig. 1 is attached to the free end of the upper strand.

[3] A. Ashkin, Acceleration and Trapping of Particles by Radiation Pressure, Phys. Rev. Lett. 24, 156 (1970).

[4] M. Bercy, Structures secondaires dans l'ARN: une étude par mesure de forces sur molécules uniques, Ph.D. thesis, PSL Research University, 2015.

[5] L. Melkonyan, Early stages of ribosome assembly, studied by single-molecule force measurements, Ph.D. thesis, PSL Research University, 2018.

[6] S. Gotta, O. Miller, and S. French, rRNA transcription rate in escherichia coli, J. Bacteriol. 173, 6647 (1991).

[7] L. Melkonyan, M. Bercy, T. Bizebard, and U. Bockelmann, Overstretching double-stranded RNA, double-stranded DNA, and RNA-DNA duplexes, Biophys. J. 117, 509 (2019).
[8] J. F. Léger, G. Romano, A. Sarkar, J. Robert, L. Bourdieu, D. Chatenay, and J. F. Marko, Structural Transitions of a Twisted and Stretched DNA Molecule, Phys. Rev. Lett. 83, 1066 (1999).

[9] T. Strick, J.-F. Allemand, D. Bensimon, A. Bensimon, and V. Croquette, The elasticity of a single supercoiled DNA molecule, Science 271, 1835 (1996).

[10] I. De Vlaminck and C. Dekker, Recent advances in magnetic tweezers, Ann. Rev. Biophys. 41, 453 (2012).

[11] D. Nelson, Statistical physics of unzipping DNA, in Forces, Growth and Form in Soft Condensed Matter: At the Interface between Physics and Biology, edited by A. T. Skjeltorp and A. V. Belushkin (Springer Netherlands, Dordrecht, 2005), pp. 65-92.

[12] H. Barkhausen, Zwei mit Hilfe der neuen Verstärker entdeckte Erscheinungen, Phys. Z. 20, 401 (1919).

[13] J. P. Sethna, K. A. Dahmen, and C. R. Myers, Crackling noise, Nature (London) 410, 242 (2001).

[14] P. L. Doussal, K. J. Wiese, S. Moulinet, and E. Rolley, Height fluctuations of a contact line: A direct measurement of the renormalized disorder correlator, Europhys. Lett. 87, 56001 (2009). 
[15] B. Gutenberg and C. Richter, Earthquake magnitude, intensity, energy, and acceleration, Bull. Seismol. Soc. Am. 46, 105 (1956).

[16] G. Blatter, M. Feigel'man, V. Geshkenbein, A. Larkin, and V. Vinokur, Vortices in high-temperature superconductors, Rev. Mod. Phys. 66, 1125 (1994).

[17] R. Burridge and L. Knopoff, Model and theoretical seismicity, Bull. Seismol. Soc. Am. 57, 341 (1967).

[18] B. Alessandro, C. Beatrice, G. Bertotti, and A. Montorsi, Domain-wall dynamics and Barkhausen effect in metallic ferromagnetic materials. I. Theory, J. Appl. Phys. 68, 2901 (1990).

[19] B. Alessandro, C. Beatrice, G. Bertotti, and A. Montorsi, Domain-wall dynamics and Barkhausen effect in metallic ferromagnetic materials. II. Experiments, J. Appl. Phys. 68, 2908 (1990).

[20] T. Nattermann, S. Stepanow, L.-H. Tang, and H. Leschhorn, Dynamics of interface depinning in a disordered medium, J. Phys. II (France) 2, 1483 (1992).

[21] O. Narayan and D. S. Fisher, Threshold critical dynamics of driven interfaces in random media, Phys. Rev. B 48, 7030 (1993).

[22] H. Bucheli, O. S. Wagner, V. B. Geshkenbein, A. I. Larkin, and G. Blatter, $(4+N)$-dimensional elastic manifolds in random media: A renormalization-group analysis, Phys. Rev. B 57, 7642 (1998).

[23] P. Chauve, P. Le Doussal, and K. J. Wiese, Renormalization of Pinned Elastic Systems: How Does It Work Beyond One Loop? Phys. Rev. Lett. 86, 1785 (2001).

[24] P. Le Doussal, K. J. Wiese, and P. Chauve, 2-loop functional renormalization group analysis of the depinning transition, Phys. Rev. B 66, 174201 (2002).

[25] P. Le Doussal, K. J. Wiese, and P. Chauve, Functional renormalization group and the field theory of disordered elastic systems, Phys. Rev. E 69, 026112 (2004).

[26] We show below that the signal specified in Eq. (7) is the same as that obtained by supposing a random force.

[27] The stiffness per trap is about $250 \mathrm{pN} / \mu \mathrm{m}$ [4], leading to half this value for the two traps. At the plateau start, the strands reduce this to $m^{2}=55 \pm 5 \mathrm{pN} / \mu \mathrm{m}$, see Fig. 1 .

[28] P. Le Doussal and K. J. Wiese, Driven particle in a random landscape: disorder correlator, avalanche distribution and extreme value statistics of records, Phys. Rev. E 79, 051105 (2009).
[29] P. Le Doussal, Finite temperature functional RG, droplets and decaying Burgers turbulence, Europhys. Lett. 76, 457 (2006).

[30] P. Le Doussal and K. J. Wiese, How to measure functional RG fixed-point functions for dynamics and at depinning, Europhys. Lett. 77, 66001 (2007).

[31] K. J. Wiese and P. Le Doussal, Functional renormalization for disordered systems: Basic recipes and gourmet dishes, Markov Processes Relat. Fields 13, 777 (2007).

[32] J. Zinn-Justin, Quantum Field Theory and Critical Phenomena (Oxford University Press, Oxford, 1989).

[33] S. Weinberg, The Quantum Theory of Fields (Cambridge University Press, Cambridge, 1995), Vols. 1-3.

[34] C. Domb, M. Green, and J. Lebowitz (eds.), Phase Transitions and Critical Phenomena (Academic, London, 1972-2001), Vols. 1-19.

[35] A. A. Middleton, P. Le Doussal, and K. J. Wiese, Measuring Functional Renormalization Group Fixed-Point Functions for Pinned Manifolds, Phys. Rev. Lett. 98, 155701 (2007).

[36] A. Rosso, P. Le Doussal, and K. J. Wiese, Numerical calculation of the functional renormalization group fixed-point functions at the depinning transition, Phys. Rev. B 75, 220201(R) (2007).

[37] For the noisy data at hand, this procedure is more stable than the one used in Refs. $[14,35,36]$, where $\Delta(w)$ was rescaled to have integral 1.

[38] P. Le Doussal and K. J. Wiese, Size distributions of shocks and static avalanches from the functional renormalization group, Phys. Rev. E 79, 051106 (2009).

[39] In Ref. [38] drops in position $u$ of size $S$ are considered, related via Eq. (1) to force drops as $\delta F=m^{2} S$.

[40] K. J. Breslauer, R. Frank, H. Blöcker, and L. A. Marky, Predicting DNA duplex stability from the base sequence, Proc. Natl. Acad. Sci. USA 83, 3746 (1986).

[41] N. Sugimoto, S. Nakano, M. Katoh, A. Matsumura, H Nakamuta, T. Ohmichi, M. Yoneyama, and M. Sasaki, Thermodynamic parameters to predict stability of RNA/DNA hybrid duplexes, Biochemistry 34, 11211 (1995).

[42] P. Chauve, T. Giamarchi, and P. Le Doussal, Creep and depinning in disordered media, Phys. Rev. B 62, 6241 (2000).

[43] J. M. Huguet, N. Forns, and F. Ritort, Statistical Properties of Metastable Intermediates in DNA Unzipping, Phys. Rev. Lett. 103, 248106 (2009).

[44] Note that $m^{2}$ here equals $k$ in Ref. [43], and $\rho_{m}$ here equals $n_{c}$ there. The theory for the exponent $4 / 3$ is given in Ref. [25], Eq. (4.22), setting $d=0$, i.e., $\varepsilon=4$ there. 